\title{
Affective Prime and Target Picture Processing: An ERP Analysis of Early and Late Interference Effects
}

\author{
Tobias Flaisch · Jessica Stockburger • \\ Harald T. Schupp
}

Accepted: 11 February 2008/Published online: 12 March 2008

(C) Springer Science+Business Media, LLC 2008

\begin{abstract}
Viewing emotionally arousing compared to neutral pictures is associated with differential electrophysiological activity in early ("early posterior negativity", EPN), as well as later time-windows ("late positive potential", LPP). A previous study revealed that the EPN is reduced when the preceding prime picture was emotional. The present study explored whether sequential interference effects are specific for early processing stages or extend to later processing stages. Dense sensor ERPs were measured while subjects viewed a continuous stream of pleasant, neutral, and unpleasant pictures, presented for $660 \mathrm{~ms}$ each. Previous results were replicated in that emotional pictures were associated with enlarged EPN and LPP amplitudes compared to neutral pictures. Furthermore, the EPN to emotional and neutral pictures was reduced when preceded by pleasant prime pictures. The novel finding was that emotional compared to neutral prime pictures were associated with reduced LPP amplitudes to the subsequently presented picture irrespective of its emotional valence (pleasant, neutral, unpleasant). These results demonstrate sustained interference effects in serial picture presentations discussed within a framework of resource competition among successive pictures.
\end{abstract}

Keywords Attention - Emotion - ERP - Interference . Early posterior negativity $\cdot$ Late positive potential

T. Flaisch $(\bowtie) \cdot$ J. Stockburger · H. T. Schupp

Department of Psychology, University of Konstanz,

P.O. Box D36, 78457 Konstanz, Germany

e-mail: tobias.flaisch@uni-konstanz.de

\section{Introduction}

Event-related potentials measured during picture viewing suggest the selective processing of emotional compared to neutral stimuli. In rapid serial presentation paradigms, a difference in processing emotional (pleasant and unpleasant), compared to neutral, pictures is shown by a larger early posterior negativity (EPN) developing around $150 \mathrm{~ms}$ after stimulus onset and lasting until about $300 \mathrm{~ms}[1,2]$. Subsequently, most apparent between 300 and $700 \mathrm{~ms}$ poststimulus, emotional pictures elicit an augmented late positive potential (LPP) over centro-parietal locations [3]. Furthermore, both ERP components vary as a function of emotional arousal. Specifically, the processing of highly arousing emotional pictures is associated with a more pronounced EPN and LPP compared to pictures of the same valence that are rated lower in arousal [2]. According to a biphasic motivational model of emotion $[4,5]$, the selective processing of pleasant and unpleasant cues during stimulus perception reflects their inherent salience, facilitating the organization of efficient actions to stimuli that can sustain (appetitive) and threaten (defensive) the life of the organism.

A unique feature of the rapid serial presentation technique is that it provides an opportunity to reveal enduring effects of emotional stimuli on subsequently presented pictures. Focusing on early processing $(\sim 150-300 \mathrm{~ms})$, a recent study observed that current target processing is affected not only by the emotional content of the target image but also systematically varies with the emotional content of the preceding prime picture [6]. When a prime picture was emotional (and itself eliciting enlarged EPNs), the EPN of the current target picture was reduced. This effect of an emotional prime was identical regardless of whether the target picture was pleasant, neutral, or 


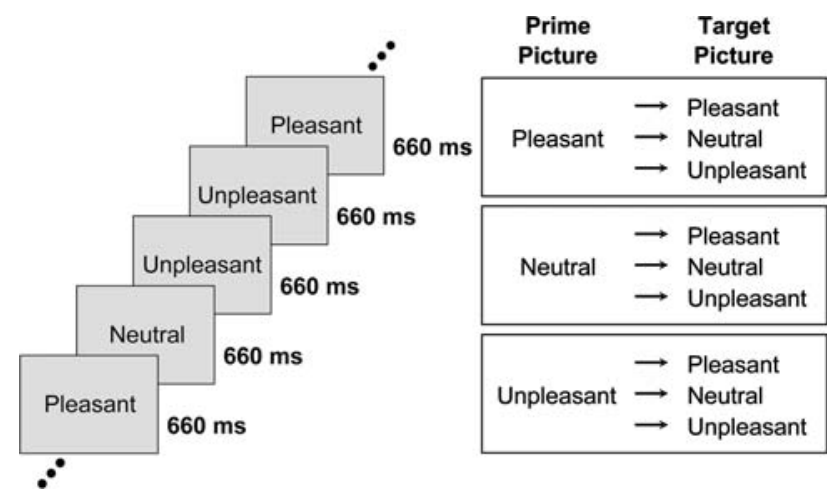

Fig. 1 Experimental design. The left panel illustrates the rapid serial visual presentation paradigm. Pleasant, neutral, and unpleasant pictures are shown for $660 \mathrm{~ms}$ without perceivable inter-stimulus gap. The right panel shows the prime-target-picture combinations examined in the present study

unpleasant. Interference at early processing stages indexed by the EPN may be related to the neural mechanism underlying the phenomenon of "emotion induced blindness' [7, 8]. Specifically, behavioral research demonstrated that explicit target picture recognition is hindered when these stimuli are preceded by emotional compared to neutral picture materials. Considered from this perspective, it is important to determine whether sequential interference effects are specific for early processing stages or extend to later processing stages, which are important for explicit stimulus recognition $[9,10]$

The present study was designed to explore the emotional modulation of the LPP as a function of the valence of the current ('target') and the preceding ('prime') picture. ${ }^{1}$ Accordingly, participants viewed a continuous stream of pleasant, neutral, and unpleasant pictures (300 pictures $\times 10$ repetitions, $660 \mathrm{~ms}$ stimulus presentation) in which the hedonic content of the current and preceding pictures varied over the course of the experiment. Pictures were presented repeatedly based on data showing that stimulus repetition does not eliminate selective processing as measured by the EPN and LPP $[11,12]$. The focus of the analysis was on ERPs to contiguous pairs of pictures in which the emotionality (pleasant, neutral, unpleasant) of each picture in the pair was systematically varied (see Fig. 1).

The main question was to determine whether the emotionality of a preceding picture impacts current picture processing as indexed by the LPP. Previous studies suggest that the LPP reflects a capacity-limited processing stage

\footnotetext{
1 The main focus in the present report is on picture pairs. In the present report, the first picture is referred to either as 'preceding' or 'prime' picture while the second picture is denoted as 'current' or 'target' picture. However, as participants viewed a continuous stream of pictures, labeling of the pictures refers to the experimental question and data analyses. Furthermore, in contrast to affective priming studies, there was no explicit task in the present study.
}

[13-16], which is related to selective attention and explicit stimulus recognition in rapid serial visual presentation paradigms [17-19]. One may therefore assume that the available resources for the current picture may vary as a function of the emotionality of the preceding picture. Specifically, emotional prime contents, which naturally draw selective attention and elicit enlarged LPP amplitudes, may reduce available resources for the picture, which is currently the focus of processing. This view predicts that emotional compared to neutral prime pictures reduce the LPP amplitudes to the current target picture irrespective of its valence. Statistically, main effects of picture emotionality for each picture in a pair (prime and target) are expected.

While congruence in hedonic valence between picture pairs has no special status in a motivated attention framework, such effects might be derived when considering the present design from the perspective of 'affective priming' [20]. Specifically, although the EPN component revealed no affective priming effects [6], such an effect may appear in later processing stages, which are related to more elaborate conceptual and semantic stimulus analysis. An affective priming hypothesis predicts an interaction between the emotionality of pictures in a pair, due to the facilitated processing of pictures that are preceded by hedonically similar pictures.

Furthermore, the present design allowed to replicate previous findings regarding prime interference effects revealed by the EPN. The crucial difference in the present compared to the previous study is presentation time (660 ms vs. $330 \mathrm{~ms}$ ). Accordingly, the present data provide first insights into the temporal sustainability of prime interference effects. Finally, using this design, we expected to replicate previous effects showing enhanced EPN and LPP amplitudes when emotional (pleasant and unpleasant), compared to neutral pictures, were the focus of current processing $[6,3]$.

\section{Materials and Methods}

\section{Participants}

Twenty-five subjects (13 female) participated in the study either for credit fulfilling an experimental participation requirement at the University of Konstanz or for monetary compensation. Participants were between the ages of 20 and 37 years $(M=24.5)$.

Stimulus Materials and Procedure

One hundred pleasant (erotic couples, babies, sports and adventure scenes), 100 neutral (neutral people, household 
objects), and 100 unpleasant pictures (mutilations, violence, attack) from the International Affective Picture System (IAPS; [21]) were presented. The picture material was the same as in Flaisch et al. [6]. The three picture categories differed significantly from each other in normative ratings of pleasure $(M=7.0,5.2$ and 2.4 for pleasant, neutral, and unpleasant contents on a 1-9 scale). Mean arousal levels for emotional categories were significantly higher than for neutral contents $(M=6.2,3.1$ and 6.4 for pleasant, neutral, and unpleasant contents, respectively). All pictures were in color and were presented with a small white fixation cross in the center. Physical parameters such as brightness, contrast and complexity did not differ across categories [1].

Pleasant, neutral, and unpleasant pictures were presented in a continuous stream without perceivable interstimulus interval for $660 \mathrm{~ms}$ each (see Fig. 1, left panel). The entire picture set was repeated 10 times resulting in a total of 3,000 picture presentations. Several constraints were imposed on the stimulus order assuring adequate control of sequence effects. Furthermore, the entire picture set was presented before the picture series was presented again in a different order. A different picture presentation order was generated for each participant.

Using Presentation software (Neurobehavioral Systems, Inc., Albany, CA), the pictures were shown on a $21 \mathrm{in.}$ CRT-monitor $(75 \mathrm{~Hz}$ refresh rate) located approximately $100 \mathrm{~cm}$ in front of the participant. Picture presentation lasted for 33 min with a short break in the middle of the session to allow for posture adjustments. Subjects were instructed to keep their eyes comfortably focused on the fixation cross in the center of the screen, and to simply view the pictures.

\section{Apparatus and Data Analysis}

Brain and ocular scalp potential fields were measured with a 256-lead geodesic sensor net (GSN 200 v2.0; EGI: Electrical Geodesics, Inc., Eugene, OR), on-line bandpass filtered from 0.01 to $100 \mathrm{~Hz}$, and sampled at $250 \mathrm{~Hz}$ using Netstation acquisition software and EGI amplifiers. Electrode impedance was kept below $50 \mathrm{k} \Omega$, as recommended for this type of electroencephalogram (EEG) amplifier by EGI guidelines. Data were recorded continuously with the vertex sensor as reference electrode. Continuous EEG data were low-pass filtered at $35 \mathrm{~Hz}$ using a zero-phase forward and reverse digital filter before stimulus synchronized epochs were extracted.

Data editing and artifact rejection were based on a twostep method for statistical control of artifacts [22]. In a first pass of the data (using the recording reference), sensors contaminated across the session were identified and rejected. Furthermore, sensors containing trial epochs with artifact activity were rejected to avoid contamination when converting the data to an average reference. The rejection of artifact-contaminated trials and sensor epochs was based on the thresholds for a number of statistical parameters (e.g., absolute value over time, standard deviation over time, etc.; [22]). In a second pass, based on the average referenced data, sensors containing artifact-contaminated activity were replaced using spherical interpolation on the basis of all remaining sensors for the given trial. Average waveforms were calculated for each of the nine experimental cells (three picture categories for the first and second picture, respectively) for each sensor and participant (see Fig. 1, right panel).

\section{Baseline Calculation}

The rapid serial visual presentation paradigm prompts issues regarding the appropriate baseline. In the current study, the use of a baseline epoch which represented all possible picture contents is perhaps the best estimate, as effects due to picture content were experimentally controlled [6]. Accordingly, in one stream of analysis, the ERP data were baseline-corrected for pre-stimulus (100 ms) prime ERP activity (first picture). However, a baseline time-locked to the target picture mathematically removes pronounced baseline-differences preceding the target picture and therefore provided a complementary view on prime effects. Thus, the data were re-analyzed in a second stream of analysis using a baseline time-locked to the onset of the target (second) picture.

\section{Waveform Analyses}

To determine critical time windows and sensor clusters sensitive to Prime and Target Valence and their interaction, exploratory waveform analyses were conducted in which each time point and sensor was tested separately using a 3 (Prime: pleasant, neutral, unpleasant) $\times 3$ (Target: pleasant, neutral, unpleasant) ANOVA [6].

\section{Early Posterior Negativity}

Early emotional modulation as a function of prime and target valence indicated by the EPN was observed in bilateral clusters over temporo-occipital as well as frontal regions. However, exploring frontal sensor sites mirrored the effects observed for the occipital negativity. For brevity, these effects are only reported in complementary analyses (see footnotes 2 and 3). Thus, for statistical analysis, the EPN amplitude was scored as mean activity in the time interval from 180 to $260 \mathrm{~ms}$ post-target picture onset and collapsed over temporo-occipital sensors with EGI sensor numbers $146,147,135,136,123,124,137,115,116,125,126,117$, 
and 118 on the left, and sensors $166,157,167,158,168,149$, $159,150,169,139,160,151$ and 140 on the right hemisphere. The EPN amplitude was submitted to repeated-measures analysis of variance (ANOVA) including the factors Prime Valence (pleasant versus neutral versus unpleasant), Target Valence (pleasant versus neutral versus unpleasant), and Laterality (left versus right).

\section{Late Positive Potential}

The LPP amplitude was derived as the mean voltage in the time interval of 520-660 ms post-target picture onset and collapsed over a centro-parietal sensor cluster containing channels with EGI sensor numbers 100, 88, 131, 78, 89, 144, 79, 132, 80, 52, 44, 257, 133 and 145. The LPP amplitude was submitted to repeated measures ANOVA with the factors Prime Valence and Target Valence.

In preliminary analysis, the EPN and LPP modulation was explored including the additional factor Gender. However, none of these analyses revealed a significant interaction involving Gender.

For effects involving repeated measures, the Greenhouse-Geisser epsilon was utilized where appropriate to correct for violations of sphericity.

\section{Results}

\section{Early Posterior Negativity (EPN)}

Figure 2 shows representative left and right occipital sensors displaying the typical finding of enlarged EPN amplitudes elicited by emotional prime pictures, accentuated for pleasant cues (upper panel). Furthermore, pleasant prime pictures reduced the EPN to the subsequent picture (target) irrespective of hedonic valence (middle panel) and this effect is accentuated when calculating a pre-target baseline (lower panel). Displaying scalp difference maps (emotional-neutral) for the EPN effect, Fig. 3 shows that prime and target effects appear with opposite polarity for pleasant cues, while unpleasant pictures do not show a consistent prime effect. These observations were supported by statistical analysis.

A first analysis was based on the baseline ERP activity preceding the prime picture. This baseline included all possible picture contents with the same probability providing a conservative assessment of ERP baseline activity. Repeated measures ANOVA revealed that the EPN varied as a function of both prime and target picture category, Prime Valence, $F(2,48)=16.0, p<0.001, \varepsilon=0.98$, and Target Valence, $F(2,48)=66.6, p<0.001, \varepsilon=0.94$, while the interaction between Prime and Target Valence was not significant, $F(4,96)=1.1$, ns.
Early Posterior Negativity Pre-Prime Baseline


Pre-Target Baseline


Fig. 2 EPN component. Illustration of the ERP waveforms for a left and right occipital sensor (EGI \#124 and \#159). The upper panel illustrates the modulation of the EPN as a function of prime picture valence. The middle panel shows the main effect of prime picture valence on the subsequent target picture processing by collapsing ERP waveforms across pleasant, neutral, and unpleasant target pictures. The lower panel shows the appearance of the prime picture effect when using a pre-target ERP baseline. Abbreviations P, N, U refer to pleasant, neutral, and unpleasant picture contents

Decomposing the main effect Target Valence replicated results from previous studies [6]. Post-hoc tests reveal that both, pleasant and unpleasant compared to neutral target pictures were associated with increased EPN amplitudes compared to neutral pictures, $F(1,24)=169.9$ and 42.9, $p<0.001$, respectively. Furthermore, as in previous research, this effect was stronger for pleasant than for unpleasant target stimuli, $F(1,24)=17.5, p<0.001$.

Decomposing the main effect Prime Valence replicated the previous finding that pleasant prime pictures profoundly affect the processing of subsequently presented pictures. The EPN measured during target presentation is reduced when preceded by a pleasant prime picture compared to both, unpleasant and neutral prime pictures, $F(1,24)=26.3$ and 19.3, $p<0.001$, respectively. Unlike 
Early Posterior Negativity

Pleasant-Neutral Unpleasant-Neutral


Fig. 3 Target picture processing. Scalp difference maps (pleasantneutral) and (unpleasant-neutral) for the EPN component (180$260 \mathrm{~ms}$ ) reveal the topography of emotion main effects on the processing of a target picture as a function of target and prime picture valence. The target valence effect is illustrated by collapsing across prime picture valence (upper row), while the prime valence effect is shown by collapsing across target picture valence (lower row). Illustration refers to the first analysis utilizing the pre-prime baseline correction. To derive these brain maps, voltages were interpolated to the scalp surface using spherical splines and back-projected to a back view of the model head. Please note the different scales for target and prime effect

previous findings [6], unpleasant prime pictures did not reliably modulate the EPN in the target window when compared to neutral cues, $F(1,24)=0.2$, ns.

Furthermore, a significant interaction between Target Valence and Laterality was observed, $F(2,48)=8.1$, $p<0.001, \varepsilon=0.96$. However, this interaction could not be meaningfully accounted for, neither by further statistical analysis, nor by visual inspection of the observed result patterns. $^{2}$

A second analysis was based on the baseline ERP activity preceding the target picture. This baseline eliminates all residual ERP differences associated with differential emotion processing. Again, significant main

\footnotetext{
$\overline{2}$ Statistical analysis of frontal sites [6] revealed a significant interaction of Prime Valence $\times$ Target Valence $\times$ Laterality, $F(4,96)=3.6, p<0.01, \varepsilon=0.9$. However, decomposing this interaction for left and right hemisphere frontal clusters yielded corresponding but polarity reversed result patterns as seen over posterior sites. Specifically, highly significant main effects for Prime Valence and Target Valence (Left: $F(2,48)=8.6$ and 33.4, $p<0.001, \varepsilon=0.97$ and $=0.95$; Right: $F(2,48)=8.8$ and 33.2, $p<0.001, \varepsilon=0.88$ and $=0.85$ ) were apparent while the interaction between Prime and Target Valence failed to reach significance on both sides, $F(4,96)=1.0$ and 1.5 , ns.
}

effects of Prime Valence and Target Valence were observed, $F(2,48)=39.9$ and 83.9, $p<0.001, \varepsilon=0.99$ and 0.97 , respectively, while the interaction of both factors was not significant, $F(4,96)=1.7$, ns. Effects regarding target valence were fully replicated. As shown in Fig. 2, lower panel, prime effects appeared more accentuated in this analysis. The EPN elicited by the target picture was reduced when preceded by pleasant compared to neutral or unpleasant prime pictures, $F(1,24)=55.0$ and 61.7 , $p<0.001$, which did not differ from each other, $F(1$, 24) $=0.1$, ns. $^{3}$

\section{Late Positive Potential (LPP)}

Figure 4 shows a representative centro-parietal sensor replicating enlarged LPP amplitudes to emotional prime pictures (upper panel). Of most interest, pleasant and unpleasant prime pictures reduced the LPP to the subsequent picture (target) irrespective of hedonic valence (middle panel). Eliminating sustained LPP effects elicited by the prime picture by calculating a pre-target baseline (lower panel), the prime effect appeared greatly enlarged. Displaying scalp difference maps (emotional-neutral) for the prime and target LPP effect, Fig. 5 reveals that the prime effect was opposite in direction to the target effect: whereas a relative positive potential over centro-parietal sites was obtained when viewing emotionally arousing first (prime) pictures, these pictures elicited a relatively more negative potential when a subsequent (target) picture was presented. These observations were supported by statistical analysis.

A first analysis was based on the baseline ERP activity preceding the prime picture. Replicating previous results, a highly significant main effect of Target Valence was obtained, $F(2,48)=40.3, p<0.001, \varepsilon=0.85$. Both, pleasant and unpleasant target pictures elicited enlarged LPP amplitudes compared to neutral target pictures, $F(1,24)=86.8$ and $40.8, p<0.001$. Furthermore, pleasant and unpleasant target pictures elicited comparable LPP amplitudes, $F(1,24)=0.1$, ns.

Of main interest with regard to prime effects on the LPP, a significant main effect of Prime Valence was observed, $F(2,48)=14.4, p<0.001, \varepsilon=0.87$, while no interaction of Prime Valence and Target Valence was apparent, $F(4,96)=1.6$, ns. Post-hoc tests reveal that pleasant and unpleasant compared to neutral prime pictures reduce the LPP to the subsequently presented picture irrespective of their hedonic valence, $F(1,24)=25.4$ and 16.2, $p<0.001$. Furthermore, when contrasting the two emotional prime

\footnotetext{
${ }^{3}$ Calculating this analysis for frontal sites [6] also yielded polarity reversed results, i.e., highly significant main effects of Prime and Target Valence, $F(4,96)=29.4$ and 53.5, $p<0.001, \varepsilon=0.91$ and 0.9 , and no significant interaction between Prime and Target Valence, $F(4,96)=1.2$, ns.
} 


\section{Late Positive Potential}

Pre-Prime Baseline
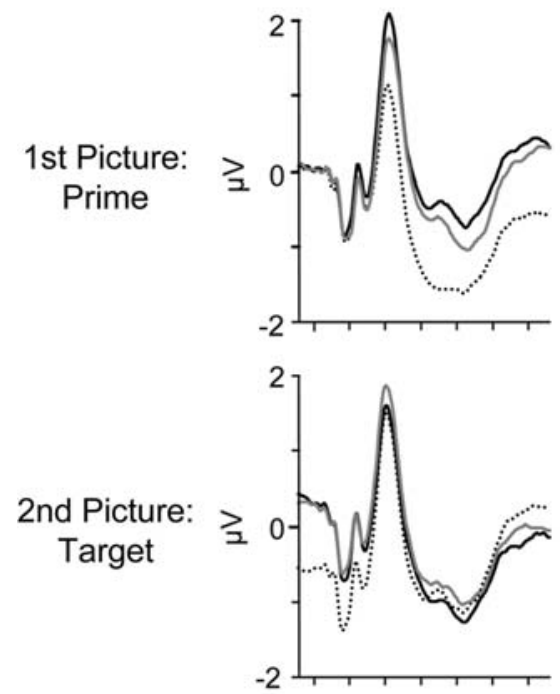

Pre-Target Baseline

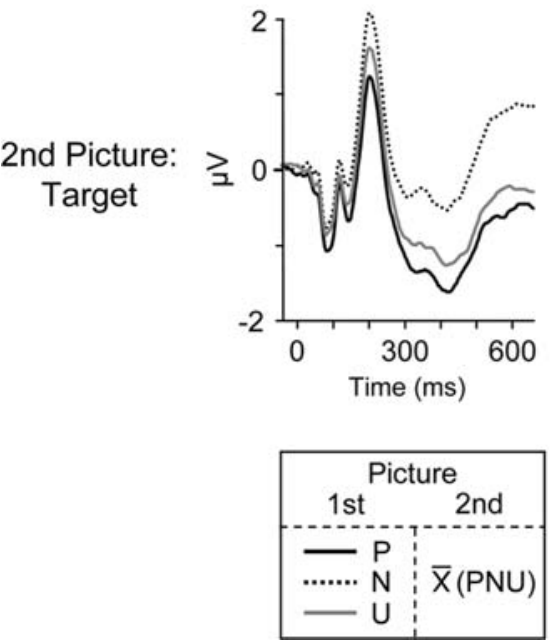

Fig. 4 LPP component. Illustration of the ERP waveforms for a representative centro-parietal sensor (EGI \#80). The upper panel illustrates the modulation of the LPP as a function of prime picture valence. The middle panel shows the main effect of prime picture valence on the subsequent target picture processing by collapsing ERP waveforms across pleasant, neutral, and unpleasant target pictures. The lower panel shows the appearance of the LPP prime picture effect when using a pre-target ERP baseline. Abbreviations P, $\mathrm{N}$, U refer to pleasant, neutral, and unpleasant picture contents

categories against each other, no significant differences were found, $F(1,24)=3.7$, ns.

A second analysis was based on the baseline ERP activity preceding the target picture. Again, significant main effects of Prime Valence and Target Valence were observed, $F(2,48)=62.2$ and $44.4, p<0.001, \varepsilon=0.88$ and $=0.79$, respectively, while the interaction of both factors was not significant, $F(4,96)=2.2$, ns. Effects regarding target valence were fully replicated. Furthermore, prime effects

\section{Late Positive Potential}

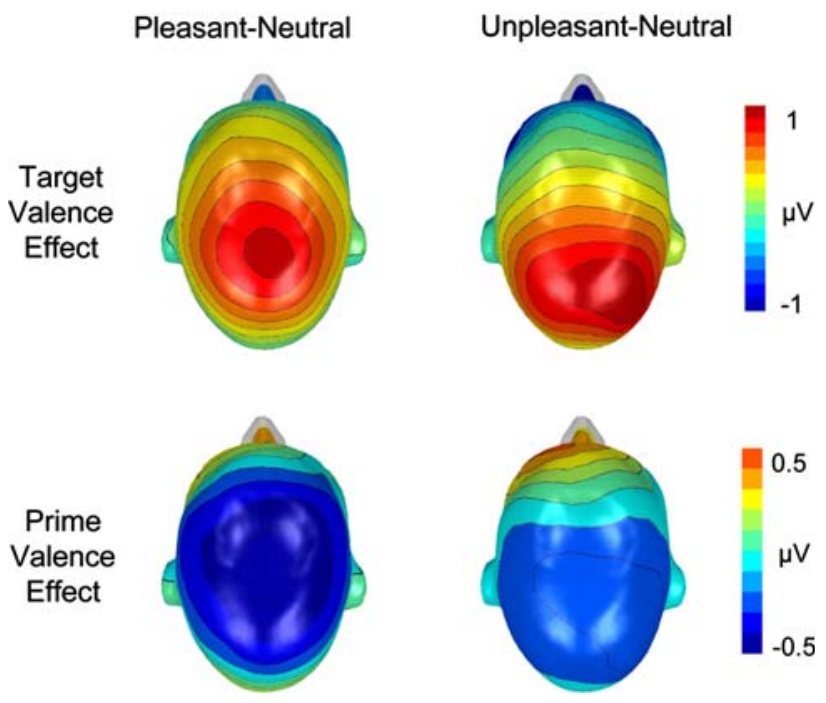

Fig. 5 Target picture processing. Scalp difference maps (emotionalneutral) for the LPP component (520-660 ms) reveal the topography of emotion main effects on the processing of a target picture as a function of target and prime picture valence. The target valence effect is illustrated by collapsing across prime picture valence (upper row), while the prime valence effect is shown by collapsing across target picture valence (lower row). Illustration refers to the first analysis utilizing the pre-prime baseline correction. A top view of the model head is shown. Please note the different scales for target and prime effect

were pronouncedly enlarged as the sustained LPP observed to emotional prime pictures reduced prime interference effects in the first analysis. Accordingly, pronouncedly reduced LPPs were elicited by target pictures when preceded by pleasant and unpleasant compared to neutral prime pictures, $F(1,24)=95.6$ and $64.4, p<0.001$, which did not differ from each other, $F(1,24)=1.8$, ns.

\section{Discussion}

Previous studies utilizing rapid picture presentations have found that emotionally significant stimuli consistently modulate both an early, as well as a late ERP component [1, $3]$. These results were replicated in the present study: pictures that were pleasant or unpleasant prompted greater EPN and LPP amplitudes compared to neutral pictures. Furthermore, the present study replicated previous findings in that the magnitude of posterior negativity was reduced when the preceding prime picture showed pleasant pictures. More importantly, the present study provides the novel finding that the amplitude of the LPP to a current target picture systematically varies with the emotional content of the preceding prime picture. When a preceding picture was emotional, the LPP was reduced to the subsequent picture irrespective of its emotional valence (pleasant, neutral, unpleasant). These 
findings are consistent with the notion that emotional cues naturally draw selective attention, which in the case of rapid serial presentations, may interfere with subsequent stimulus processing in capacity-limited processing stage indexed by the LPP [18, 17]. Overall, the EPN and LPP findings may provide ERP evidence for the finding that the detection of target stimuli suffers from following an affectively engaging picture [7, 8], presumably reflecting competition for processing resources among successive picture presentations.

The main finding of the present study was that the LPP elicited by pleasant, neutral, and unpleasant pictures was reduced when preceded by an emotional picture, which itself elicit enlarged LPPs compared to neutral images. As rapid picture presentations may raise concerns regarding baseline activity, it is noteworthy that conceptually similar results were observed for both streams of analysis. Specifically, while a baseline locked to the target picture may overestimate prime effects, a baseline preceding the prime pictures may underestimate prime effects as neutral pictures elicit a pronouncedly reduced LPP compared to emotional pictures (see Fig. 3). Accordingly, while there may be no optimal measure of baseline activity in the rapid picture presentation paradigm, there is converging evidence that emotional prime pictures reduce the LPP elicited by subsequent pictures across both measures of ERP baseline activity.

One perspective to consider the present LPP findings is the notion of competition and sharing of processing resources, which is often invoked in behavioral research relying on rapid serial presentation paradigms [23, 24]. Previous studies already suggested that late positive potentials in this time window reflect the operation of a capacity-limited processing system. Most relevant, dual-task studies have consistently revealed a reduced P3 wave to a target stimulus in a secondary (oddball) task when attention was directed to a primary task in the visual modality [25]. These results were obtained with non-obtrusive tones as well as salient startle stimuli $[13,14]$. The present findings extend these data by showing similar effects in natural selective attention paradigms, in which no explicit task focus is demanded. Similarly, the startle probe methodology has been used to show that variations in natural selective attention interfere with concurrent stimulus processing as measured by the P3 amplitude to salient startle tones. Specifically, when viewing emotional, compared to neutral, pictures, the obligatory startle P3 was reduced [16, 26]. In this regard, the present findings are novel by showing that effects of interference by emotional prime pictures extend in time and affect the processing of subsequent stimuli in the rapid serial presentation paradigm [27]. Taken together, the present study suggests that emotional pictures interfere with subsequent stimulus processing in a capacity-limited processing stage indicated by the LPP, which has been implicated in focused attention and conscious stimulus recognition.
From a functional perspective, a considerable amount of research relates late positive potentials to the quality and accuracy of stimulus processing. For instance, research using stimulus recognition tasks revealed that reported confidence in recognition is associated with increased P3 amplitudes [28]. Furthermore, attentional blink studies demonstrate that the P3 amplitude systematically varied with conscious recognition [17]. Specifically, seen target stimuli (presented during the attentional blink interval) elicited a P3 wave, which was absent for unseen target stimuli [18, 19]. In addition, findings from a masking study suggest the P3 as neural correlate of conscious recognition reflecting widespread activity in fronto-parieto-temporal networks [19]. Based on these data, one may speculate that a reduced LPP to target pictures, due to a preceding emotional prime picture, reflects a loss in the quality and accuracy of conscious stimulus recognition. In a series of three studies, we provide behavioral support for this notion by showing that emotional, compared to neutral, pictures interfered with stimulus recognition of subsequently presented neutral stimulus materials (Flaisch et al., submitted).

The LPP is preceded by a transitory ERP component, the EPN, which is also sensitive to the emotional significance of pictures. In a previous study, the EPN was subject to interference by emotional prime pictures. Specifically, the EPN elicited by target pictures over posterior sensor locations was reduced when preceded by pleasant and unpleasant compared to neutral cues [6]. These findings were replicated with regard to pleasant pictures. In contrast, unpleasant prime pictures failed to show interference effects when compared to neutral cues in the present study. Several aspects may contribute to the different findings regarding unpleasant pictures across both studies. The main difference between both studies is presentation time (330 ms vs. $660 \mathrm{~ms}$ ). It may be hypothesized that processing interference in fleeting processing stages resolves with increasing processing times. Accordingly, EPN prime effects may be less pronounced in the present study because of longer picture presentations. Consistent with this notion, prime interference effects were diminished in the present compared to the previous study for unpleasant $(0.02 \mu \mathrm{V}$ vs. $0.28 \mu \mathrm{V})$ and pleasant $(0.34 \mu \mathrm{V}$ vs. $0.51 \mu \mathrm{V})$ cues. Furthermore, the finding that pleasant prime pictures elicited significant target interference effects seems to be secondary to greater EPN modulation for pleasant cues, resulting in more pronounced prime interference to emotional and neutral target pictures. Previous findings suggest that the pleasant and unpleasant EPN difference is mediated primarily by the great number of pictures with erotic content [11]. Taken together, the EPN prime effect for high-arousing pleasant cues with longer presentation times (660 ms) suggest that interference effects extend in time possibly due to reentrant activation of temporo-occipital 
regions implicated in visual stimulus processing. To more precisely explore the temporal dynamics of affective interference effects, future research may systematically vary picture presentation times.

In the present study, neither the EPN nor the LPP component were affected by the congruency in hedonic valence between picture pairs, i.e., whether prime and target picture are similar or different in hedonic valence. These findings contrast with previous ERP studies exploring effects associated with stimulus repetitions in affective priming and oddball-like experimental designs using emotional facial expressions [29, 30]. However, pronounced differences in terms of experimental design and emotional stimulus materials render a comparison of these and the present study difficult. With regard to experimental methods, a major difference concerns task set. The present research relied on passive picture viewing while different result patterns might emerge in active task contexts. With regard to emotional stimulus materials, IAPS pictures of high emotional intensity were used, which seemingly engage emotional response channels to a much higher degree than facial expressions [31]. It therefore seems noteworthy that despite presenting highly arousing stimuli no reliable hedonic congruency effects between picture pairs were observed. The inclusion of neutral stimulus materials seems to be helpful to empirically compare the notion of interference and affective priming. Overall, it seems important to determine in future studies whether affective priming effects are observed with IAPS picture materials in active task contexts.

A large array of studies including behavioral, neuroimaging, and psychophysiological measures suggest that emotional pictures naturally draw selective attention [5]. The rapid serial presentation paradigm affords to explore how picture processing varies as a function of previously seen emotional and neutral pictures. Focusing on late positive potentials, the present findings demonstrate that emotionally arousing pictures affect the processing of subsequently presented pictures, irrespective of whether these pictures depicted emotional or neutral contents. These findings are consistent with the notion of interference and competition for processing resources suggested by cognitive and neuroscientific studies [23, 32-34].

Acknowledgments This research was supported by grants from the German research foundation Schu1074/10-1 and Schu1074/7-4 to Harald Schupp.

\section{References}

1. Junghöfer M, Bradley MM, Elbert TR, Lang PJ. Fleeting images: a new look at early emotion discrimination. Psychophysiology 2001;38:175-8.
2. Schupp HT, Junghöfer M, Weike AI, Hamm AO. Emotional facilitation of sensory processing in the visual cortex. Psychol Sci 2003;14:7-13.

3. Schupp HT, Stockburger J, Codispoti M, Junghöfer M, Weike AI, Hamm AO. Selective visual attention to emotion. J Neurosci 2007;27:1082-9.

4. Lang PJ, Bradley MM, Cuthbert BN. Motivated attention: affect, activation, and action. In: Lang PJ, Simons RF, Balaban M, editors. Attention and emotion: sensory and motivational processes. Mahwah, NJ: Erlbaum; 1997. pp 97-135.

5. Bradley MM, Lang PJ. Emotion and motivation. In: Cacioppo JT, Tassinary LG, Berntson GG, editors. Handbook of psychophysiology. New York: Cambridge University Press; 2000. pp 60242.

6. Flaisch T, Junghöfer M, Bradley MM, Schupp HT, Lang PJ. Rapid picture processing: affective primes and targets. Psychophysiology 2008;45:1-10.

7. Most SB, Chun MM, Widders DM, Zald DH. Attentional rubbernecking: cognitive control and personality in emotion-induced blindness. Psychon Bull Rev 2005;12:654-61.

8. Most SB, Smith SD, Cooter AB, Levy BN, Zald DH. The naked truth: positive, arousing distractors impair rapid target detection. Cogn Emot 2007;21:964-81.

9. Schupp HT, Flaisch T, Stockburger J, Junghöfer M. Emotion and attention: event-related brain potential studies. Prog Brain Res 2006;156:31-51.

10. Del Cul A, Baillet S, Dehaene S. Brain dynamics underlying the nonlinear threshold for access to consciousness. PLoS Biol 2007;5:2408-23.

11. Codispoti M, Ferrari V, Bradley MM. Repetitive picture processing: autonomic and cortical correlates. Brain Res 2006;1068:213-20.

12. Codispoti M, Ferrari V, Bradley MM. Repetition and eventrelated potentials: distinguishing early and late processes in affective picture perception. J Cogn Neurosci 2007;19:577-86.

13. Isreal JB, Chesney GL, Wickens CD, Donchin E. P300 and tracking difficulty: evidence for multiple resources in dual-task performance. Psychophysiology 1980;17:259-73.

14. Roth WT, Dorato KH, Kopell BS. Intensity and task effects on evoked physiological response to noise bursts. Psychophysiology 1984;21:466-81.

15. Polich J. P300 from a passive auditory paradigm. J Electroencephalogr Clin Neurophysiol 1989;74:312-20.

16. Schupp HT, Cuthbert BN, Bradley MM, Birbaumer N, Lang PJ. Probe P3 and blinks: two measures of affective startle modulation. Psychophysiology 1997;34:1-6.

17. Vogel EK, Luck SJ, Shapiro KL. Electrophysiological evidence for a postperceptual locus of suppression during the attentional blink. J Exp Psychol Hum Percept Perform 1998;24:1656-74.

18. Kranczioch C, Debener S, Engel AK. Event-related potential correlates of the attentional blink phenomenon. Cogn Brain Res 2003; 17:177-87.

19. Sergent C, Baillet S, Dehaene S. Timing of the brain events underlying access to consciousness during the attentional blink. Nat Neurosci 2005;8:1391-400.

20. Klauer KC, Musch J. Affective priming: findings and theories. In: Musch J, Klauer KC, editors. The psychology of evaluation: affective processes in cognition and emotion. Mahwah NJ: Erlbaum; 2003. pp 7-49.

21. Lang PJ, Bradley MM, Cuthbert BN. International affective picture system (IAPS): affective ratings of pictures and instruction manual. Technical Report A-6. University of Florida, Gainesville, FL; 2005.

22. Junghöfer M, Elbert T, Tucker DM, Rockstroh B. Statistical control of artifacts in dense array EEG/MEG studies. Psychophysiology 2000;37:523-32. 
23. Potter MC. Understanding sentences and scenes: the role of conceptual short-term memory. In: Coltheart V, editor. Fleeting memories: cognition of brief visual stimuli. The MIT Press: Cambridge, MA; 1999. pp 13-46.

24. Shapiro K, Arnell KM, Raymond JE. The attentional blink. Trends Cogn Sci 1997;1:291-6.

25. Donchin E, Kramer AF, Wickens C. Applications of brain eventrelated potentials to problems in engineering psychology. In: Coles MGH, Donchin E, Porges SW, editors. Psychophysiology: systems, processes, and applications. The Guilford Press: New York; 1986. pp 702-18.

26. Schupp HT, Cuthbert BN, Bradley MM, Hillman CH, Hamm AO, Lang PJ. Brain processes in emotional perception: motivated attention. Cogn Emot 2004;18:593-611.

27. Potter MC, Staub A, O'Connor DH. The time course of competition for attention: attention is initially labile. J Exp Psychol Hum Percept Perform 2002;28:1149-62.

28. Hillyard SA, Squires KC, Bauer JW, Lindsay PH. Evoked potential correlates of auditory signal detection. Science 1971;25:1357-60.
29. Werheid K, Alpay G, Jentzsch I, Sommer W. Priming emotional facial expressions as evidenced by event-related brain potentials. Int J Psychophysiol 2005;55:209-19.

30. Campanella S, Quinet P, Bruyer R, Crommelinck M, Guerit JM. Categorical perception of happiness and fear facial expressions: an ERP study. J Cogn Neurosci 2002;14:210-27.

31. Sabatinelli D, Bradley MM, Fitzsimmons JR, Lang PJ. Parallel amygdala and inferotemporal activation reflect emotional intensity and fear relevance. Neuroimage 2005;24:1265-70.

32. Keysers C, Perrett DI. Visual masking and RSVP reveal neural competition. Trends Cogn Sci 2002;6:120-5.

33. Pessoa L, McKenna M, Gutierrez E, Ungerleider LG. Neural processing of emotional faces requires attention. Proc Natl Acad Sci USA 2002;99:11458-63.

34. Pourtois G, Grandjean D, Sander D, Vuilleumier P. Electrophysiological correlates of rapid spatial orienting towards fearful faces. Cereb Cortex 2004;14:619-33.

35. Flaisch T, Steinhauser M, Hübner R, Schupp HT. Emotional aftereffects: when emotion impairs subsequent picture recognition; submitted. 